\title{
A MOLECULAR DYNAMICS CALCULATION TO CASCADE DAMAGE PROCESSES
}

\author{
Hiwa Mohammad QADR \\ College of Science, University of Raparin, Sulaimanyah, Iraq \\ e-mail: hiwa.physics@uor.edu.krd
}

\begin{abstract}
In this work, Molecular dynamics simulation was performed to study the cascade damage evaluation initial from a $250 \mathrm{eV}$ Primary Knock-on Atoms (PKAs) in gold. For this purpose, the simulations were carried out using the molecular dynamics code GRAPE to study the cooling phase of a cascade from the thermodynamic point view. Interatomic interaction of the cascade was investigated by the Morse potential and it is found that during the cooling phase of the cascade local equilibrium was realized.
\end{abstract}

KEYWORDS: displacement cascade, primary damage, Morse potential, molecular dynamics

\section{Introduction}

Radiation damage processes from initial defect production to microstructure evolution occur over a wide spectrum of the time and size scale. Atomic collision sequences during the collisional phase continue for a few tenth of picosecond in a small region on nanometer scale. The subsequent cooling phase takes place for nearly $10^{-11} \sec [1,2]$.

Molecular dynamics method is a well- defined microscopic description of many-body system [3]. Computing the phase space trajectories of a collection of molecules that individually obey classical laws of motion [4], molecular dynamics computer simulations provide the most realistic description of the primary damage state including the collisional and cooling phase. Binary collision approximation provides a realistic description of the gross features of defect spatial distribution with requisite physical reality in statistically significant numbers, including the size, number and spacing of subcascades [5, 6].

The cascade damage methods are classified into four stages (Thermal phase, collisional phase, diffusional phase and cooling phase). The cooling phase is defined as stage of the dissipation of energies deposited locally during the collisional phase, ending when the cascade region reaches thermal equilibrium with its surroundings. During the cooling phase, average kinetic energy in a cascade core is well above the melting temperature of materials and the significant rearrangement of defects takes place, including recombination and clustering, after the cooling phase, interaction of point defects occurs by normal, thermally activated diffusion [7].

Displacement cascade, in a material, can be visualized as a series of elastic collisions initiated with the lattice atoms, where the lattice atom is hit by a high energy particle. The initially-bombard a target is named PKA. The Norgett-Robinson-Torrens (NRT) model suggested that the number of Frenkel pairs produced by a PKA of energy [8-10]. It is used to calculate radiation damage calculation method exposure unit known as displacement per atom (dpa). Thus, dpa has been used as a standard measure for computing proton- neutron induced radiation damage production from different radiation sources [11-15]. Molecular dynamics simulation of processes in metals have been studied by many research groups including temperature dependence of the processes of cascade damage [16-18].

MDGRAPE code is a series of special-purpose computer system built for particle-based simulations such as molecular dynamics simulation [19, 20]. In this paper, the primary states of cascade damage processes are simulated to study the cooling phase in gold, using the molecular dynamics simulation method.

\section{Molecular dynamics simulation methods}

A major computer simulation method to analyse development processes of cascade damage is GRAPE code to study the cooling phase of a cascade from the 
thermodynamic point view. Molecular dynamics are performed to study a computational cell and to apply many body interatomic potential based on embedded atom method (EAM) [21] to description of atom behaviour. In this paper, $250 \mathrm{eV}$ cascade is simulated in gold. One atom is chosen as $250 \mathrm{eV}$ PKA in the direction of [120], from the computational cell. Computational cell contains 10800 gold atoms corresponding to a rectangular parallelepiped of $15 \alpha_{0}$ x $15 \alpha_{0} \times 12 \alpha_{0}$, where $\alpha_{0}$ is the lattice constant. For atoms in the vicinity of the boundary of the cell, periodic boundary conditions are employed. A time step equals to $3 \times 10^{-15} \mathrm{~s}$ for all the calculations. Since the system is thermally insulated, in the present study, interatomic interaction is described using pair- wise Morse potential [22] which is adjusted by the cohesive energy, elastic constants and compressibility of gold.

\section{Results and discussion}

In this study, Molecular dynamics measurement was performed to study the cooling phase of a cascade in gold, considering from the thermodynamic point view that the initial lattice temperature is $0 \mathrm{~K}$. Figure 1 shows the time dependence of the number of displaced atoms for $250 \mathrm{eV}$ cascade by using Morse potential where a displaced atom is defined to be displaced by over one-quarter of the lattice constant from nearest lattice site. It is also shown that an initial increase is followed by a decrease due to the spontaneous recombination of unstable defect pairs. Furthermore, durations of relaxation phases and collisional are estimated to be both 0.13 psec. It also observes the migration of a small number of interstitial atoms during the following cooling phase.

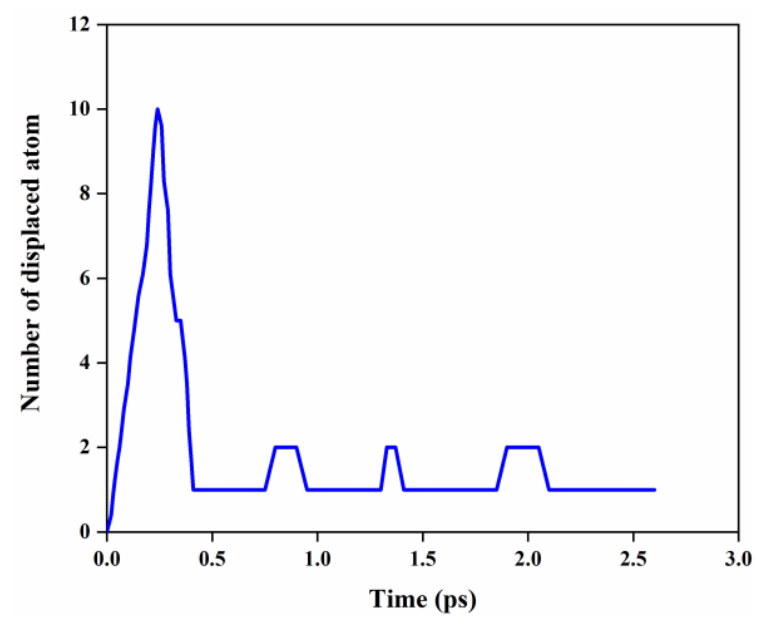

Fig. 1. Number of displaced atoms as a function of time for a $250 \mathrm{eV}$ cascade in gold using the Morse potential
Figure 2 shows the time dependence of average kinetic energy. In the collisional phase the average kinetic energy rapidly decreases due to conversion of the kinetic energy to potential energy, establishing approximate equipartition of energy during the subsequent cooling phase, the kinetic energy remains constant due to the thermally insulated system. Furthermore, during the cooling phase, the migration of interstitial atoms with relatively high kinetic energies $(0.1 \sim 1 \mathrm{eV})$ is observed.

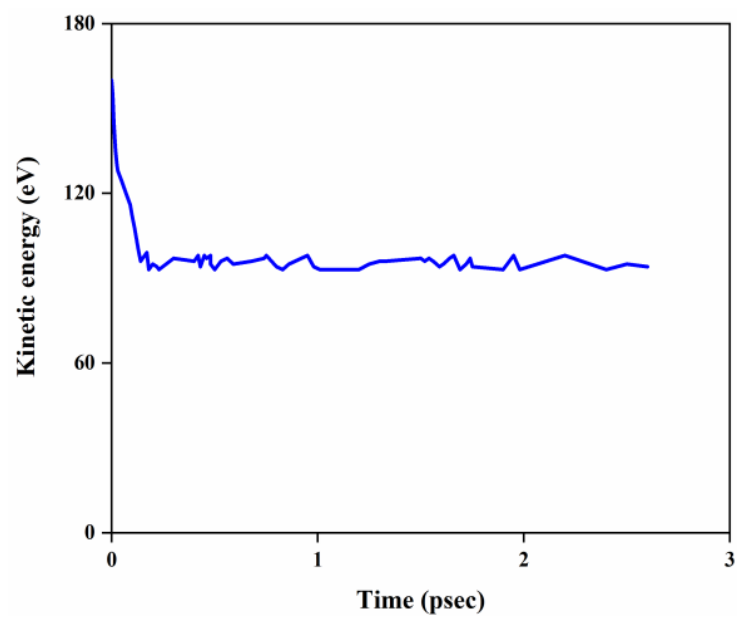

Fig. 2. Average kinetic energy as a function of time for a $250 \mathrm{eV}$ cascade in gold using the Morse potential

Figure 3 shows the velocity distribution at 0.26 , 0.52 and $2.0 \mathrm{psec}$. It is clear that the velocity distribution of moving atoms can change and gradually approaches the Maxwell Boltzmann distribution. It is observed that from the initial stage of the cooling phase, the system is strongly deviated from thermal equilibrium, while, at top stage of the cooling phase, equilibrium velocity distribution has been almost established.

The velocity distribution of the central region is almost close to the Maxwell Boltzmann distribution as shown in Figure 4. However, statistics is not enough to give a smooth curve. This result is calculated using the adiabatic molecular dynamics system where electronic contribution to dissipation of thermal energies in cascades is not considered. Since in noble metals as gold, the contribution is small due to weak coupling of the electronic system with the lattice. Thus, Figure 5 does not indicate an equilibration between the electronic and lattice systems. However, this figure seems to imply that at least, local temperature of the lattice system can be described even at such an early stage of the cooling phase of cascade evolution. 


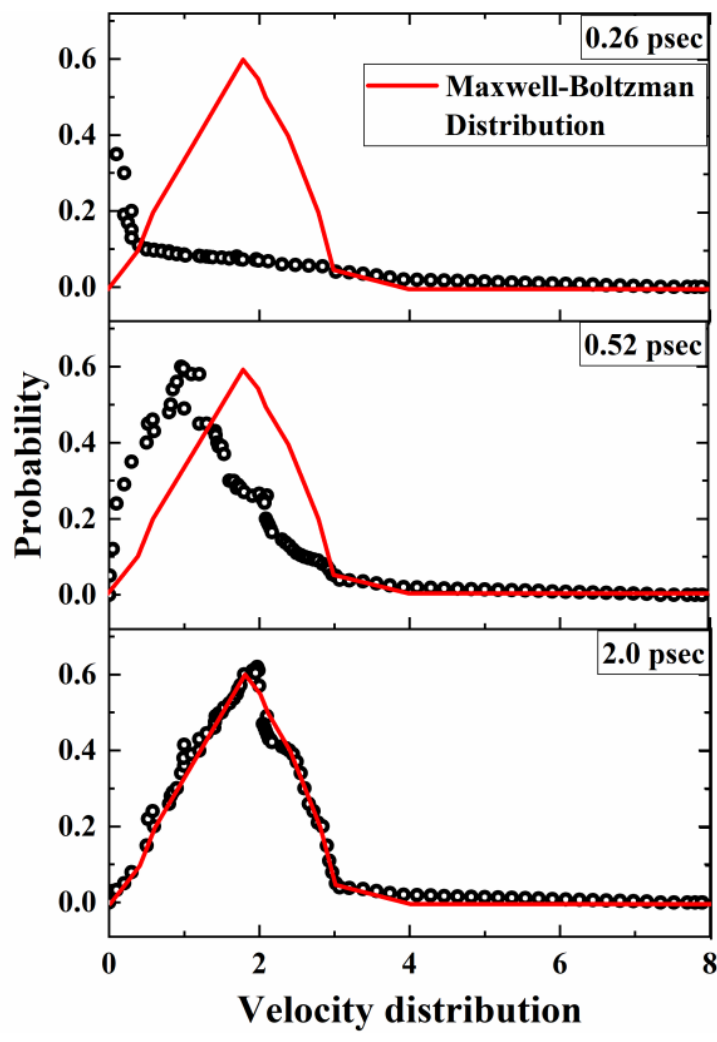

Fig. 3. Velocity distribution during the cooling phase of cascade for three different times

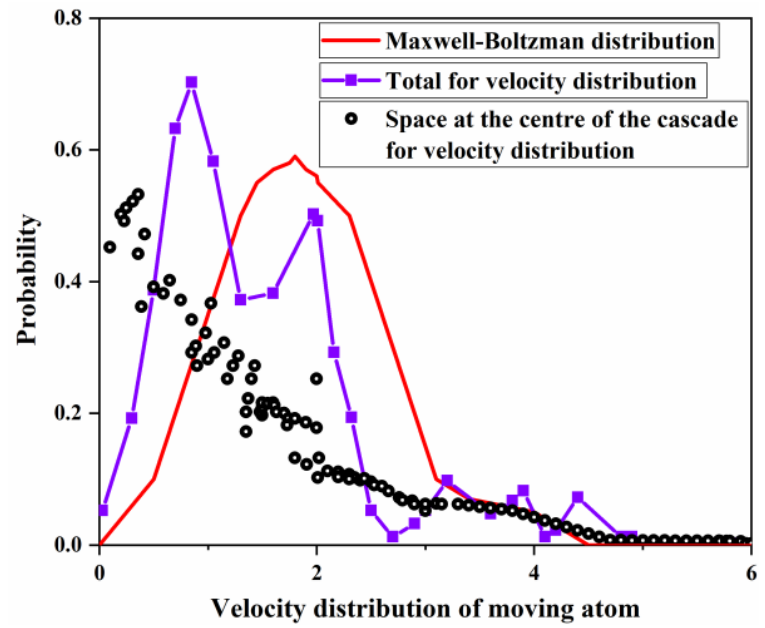

Fig. 4. A Comparison of velocity distributions during the cooling phase of cascade at $0.4 \mathrm{psec}$

\section{Conclusions}

In this study, the primary states of cascade damage processes are simulated to study the cooling phase, using the molecular dynamics calculation at $250 \mathrm{eV}$ in gold. At early stage in cascade cooling phase, the total kinetic energy remains constant due to the thermally insulated system, the velocity distribution of moving atoms can change and gradually approaches the equilibrium distribution. Local equilibrium of the lattice is established, indicating that the simple jump processes of defects may be applied to the simulation of cascade evolution after the collisional phase. Accordingly, more work could be done by using extremely high energy, temperature and more metals.

\section{References}

[1]. Nordlund K., Zinkle S. J., Sand A. E., Granberg F., Averback R. S., Stoller R. E., et al., Primary radiation damage: A review of current understanding and models, Journal of Nuclear Materials, 512, p. 450-79, 2018.

[2]. Krasheninnikov A., Nordlund K., Ion and electron irradiation-induced effects in nanostructured materials, Journal of applied physics, 107 (7), p. 3, 2010.

[3]. Zwicknagel G., Molecular dynamics simulations of the dynamics of correlations and relaxation in an $O C P$, Contributions to Plasma Physics, 39 (1-2), p. 155-8, 1999.

[4]. Heermann D. W., Computer-simulation methods. Computer Simulation Methods in Theoretical Physics, Springer, p. 8-12, 1990

[5]. Gibson J., Goland A. N., Milgram M., Vineyard G., Dynamics of radiation damage, Physical Review, 120 (4), p. 1229, 1960.

[6]. Antoshchenkova E., Luneville L., Simeone D., Stoller R. E., Hayoun M., Fragmentation of displacement cascades into subcascades: A molecular dynamics study, Journal of Nuclear Materials, 458, p. 168-75, 2015.

[7]. Bacon D. J., de la Rubia T. D., Molecular dynamics computer simulations of displacement cascades in metals, Journal of Nuclear Materials, 216, p. 275-90, 1994.

[8]. Weber W. J., Zhang Y., Predicting damage production in monoatomic and multi-elemental targets using stopping and range of ions in matter code: Challenges and recommendations, Current Opinion in Solid State and Materials Science, 23 (4), p. 100757, 2019.

[9]. Stoller R. E., Toloczko M. B., Was G. S., Certain A. G., Dwaraknath S., Garner F. A., On the use of SRIM for computing radiation damage exposure, Nuclear instruments and methods in physics research section B: beam interactions with materials and atoms, 310, p. 75-80, 2013.

[10]. Qadr H. M., Radiation damage and dpa in iron using mcnp5, European Journal of Materials Science and Engineering, 5 (3), p. 109-14, 2020.

[11]. Qadr H., Effect of Ion Irradiation on the Mechanical Properties of High and Low Copper, Atom Indonesia, 46 (1), p. 47$51,2020$.

[12]. Qadr H., Effect of ion irradiation on the hardness properties of Zirconium alloy, Annals of the University of Craiova, Physics, 29, p. 68-76, 2019.

[13]. Qadr H. M., Hamad A. M., Mechanical Properties of Ferritic Martenstic Steels: A Review, Scientific Bulletin of Valahia University-Materials and Mechanics, 17 (16), p. 18-27, 2019.

[14]. Hiwa M., Ari M., Investigation of long and short term irradiation hardening of P91 and P92 ferritic/martensitic steels, Вопросы атомной науки и техники Серия: Термоядерный синтез, 42 (2), p. 81-8, 2019.

[15]. Hiwa M., Stopping power of alpha particles in helium gas, Вестник Московского государственного технического университета им НЭ Баумана Серия «Естественные науки», 2 (89), p. 117-25, 2020.

[16]. Arouche TdS., Cavaleiro R. MdS., Tanoue P. S. M., Pereira T. Sd. S., Neto A. Md. J. C., Heavy Metals Nanofiltration Using Nanotube and Electric Field by Molecular Dynamics, Journal of Nanomaterials, 2020. 


\section{THE ANNALS OF “DUNAREA DE JOS" UNIVERSITY OF GALATI \\ FASCICLE IX. METALLURGY AND MATERIALS SCIENCE \\ $\mathrm{N}^{\circ} .4$ - 2020, ISSN 2668-4748; e-ISSN 2668-4756 \\ Article DOI: https://doi.org/10.35219/mms.2020.4.02}

[17]. Gaillac R., Pullumbi P., Bennett T. D., Coudert F.-X., Structure of Metal-Organic Framework Glasses by Ab Initio Molecular Dynamics, Chemistry of Materials, 2020.

[18]. Zheng F., Wang L.-W., Exploring non-adiabaticity to $C O$ reduction reaction through ab initio molecular dynamics simulation, APL Materials, 8 (4), p. 041115, 2020.

[19]. Ohmura I., Morimoto G., Ohno Y., Hasegawa A., Taiji M., MDGRAPE-4: a special-purpose computer system for molecular dynamics simulations, Philosophical Transactions of the
Royal Society A: Mathematical, Physical and Engineering Sciences, 372 (2021), p. 20130387, 2014.

[20]. Sheel T., Yasuoka K., Obi S., Fast vortex method calculation using a special-purpose computer, Computers \& fluids, 36 (8), p. 1319-26, 2007.

[21]. Ackland G., Tichy G., Vitek V., Finnis M., Simple N-body potentials for the noble metals and nickel, Philos Mag A, 56 (6), p. 735-56, 1987.

[22]. Torrens I., Interatomic potentials, Elsevier, 2012. 